\title{
A statistical approach to map matching using road network geometry, topology and vehicular motion constraints
}

\author{
Oliver Pink, Britta Hummel \\ Institut für Mess- und Regelungstechnik \\ Universität Karlsruhe (TH), 76128 Karlsruhe, Germany \\ \{pink, hummel\}@mrt.uka.de
}

\begin{abstract}
This paper presents a method for reliable matching of position and orientation measurements from a standard GPS receiver to a digital map. By incorporating road network topology in the matching process using a hidden Markov model, an optimum position and orientation history can be computed from a sequence of GPS measurements.

Increased robustness is achieved by introducing constraints for vehicular motion in an extended Kalman filter and by reconstructing the original road network from the digital map using cubic spline interpolation. The proposed method delivers robust matching results for standard inner-city scenarios and gives a reliable estimate of the optimal position history even for severely disturbed GPS measurements.
\end{abstract}

\section{INTRODUCTION}

Recent years have shown a tremendous increase in demand of mobile geographic information systems. Starting from built-in and hand-held navigation systems, a large number of applications in intelligent transportation systems like fleet management, road traffic management or electronic toll collection has evolved.

An essential task for these applications is the assignment of a vehicle position measurement to a position in a digital map, the so-called map matching. Many of these applications, do not only require a reliable estimate of the current vehicle position, but also an estimate of the driven path. Considering the correct charging of road tolls, posterior knowledge of the driven path is essential, while a false intermediate position estimate is tolerable.

Previous work on map matching has focused especially on fast and reliable estimation of the current vehicle position in a digital map. An overview on earlier work can be found in [1]. More recent work on map matching has focused especially on robustness and reconstruction of the vehicle path [9], [2].

In our previous work, we have already presented a method for robust, GPS only map matching [6] that has already shown promising results in determining the vehicle position history. In this paper, we will present an improved method that allows for robust estimation of the vehicle position history over long periods of time and even with severely disturbed GPS measurements.

The remainder of this paper will be organized as follows. In section II, the objective of this paper is formulated and our system setup will be introduced. In section III, filtering of the raw GPS measurements in an extended Kalman filter is described. Reconstruction of the road geometry from the digital map is explained in section IV and the representation of road topology in a hidden Markov model is presented in section V. The map matching process itself is explained in section VI and experimental results are given in section VII. Section VIII gives a summary and concludes the paper.

\section{PRELIMINARIES}

Our approach will be described in two steps: First a generic map matching problem will be formulated, and second a suitable statistical representation of the measurement uncertainties will be derived.

The generic problem can be described as finding the optimal vehicle path on a road network for a given set of vehicle position and orientation measurements with known uncertainties of both the map and the position and orientation estimates. This part will be described as a standard Bayesian classification task that is independent of the particular sensor platform chosen. Sensor characteristics are incorporated in a representation of measurement uncertainties. The sensor setup we will use only consists of a standard GPS receiver and an industry-standard digital map. We chose this setup because it is the smallest possible set of sensors that is used in practice, e.g. for portable navigation devices. The main idea is that using additional sensors or sensors with a higher precision will very likely yield better map matching results.

The GPS receiver delivers one measurement for vehicle position and orientation per second. While there is no uncertainty of the vehicle orientation available, the horizontal dilution of precision (HDOP) can be used as a coarse estimate for the position uncertainties. However, the orientation uncertainty can be modeled as inversely proportional to vehicle speed.

The digital representation of the road network is compliant to the GDF standard [7]. Road network topology is described by nodes and edges, where every edge represents one road segment and a node represents connections between those road elements. Additional properties may restrict certain driving maneuvers for both nodes and edges, such as oneway road elements or right-turn only at intersections.

Road network geometry is represented by the geographic positions of the nodes and by additional shape points that may be assigned to an edge. Shape points are usually placed to keep the lateral deviation under a certain threshold, but the exact placement is vendor-specific. However, both nodes and shape points are assumed to lie exactly on the center line of the road. 


\section{Vehicle State Estimation}

The vehicle state $\mathbf{x}$ that will be needed for the map matching algorithm consists of a 2-dimensional position estimate $[x, y]^{T}$, a heading angle estimate $\varphi$ and the corresponding uncertainties, i.e. the $3 \times 3$ covariance matrix $\boldsymbol{\Sigma}_{\mathbf{x}}$.

Instead of directly using the measurements from the GPS receiver as state estimates, we will use a simple constant velocity and constant yaw rate model in an extended Kalman filter for state estimation. The continuous-time system model is determined according to figure 1 :

$$
\left[\begin{array}{c}
\dot{x} \\
\dot{y} \\
\dot{\varphi} \\
\dot{v} \\
\dot{\eta}
\end{array}\right]=\left[\begin{array}{c}
-v \cdot \sin (\varphi) \\
v \cdot \cos (\varphi) \\
\eta \\
0 \\
0
\end{array}\right]
$$

where $x$ and $y$ are the vehicle position, $\varphi$ is the vehicle heading and $v$ is the vehicle speed. The yaw rate is denoted $\eta$ to avoid confusion with the state variable $\dot{\varphi}$.

The corresponding discrete-time approximation with sampling time $T$ is

$$
\left[\begin{array}{c}
x_{k+1} \\
y_{k+1} \\
\varphi_{k+1} \\
v_{k+1} \\
\eta_{k+1}
\end{array}\right]=\left[\begin{array}{c}
x_{k}-v_{k} \cdot T \cdot \sin \left(\varphi_{k}\right) \\
y_{k}+v_{k} \cdot T \cdot \cos \left(\varphi_{k}\right) \\
\varphi_{k}+\eta_{k} \cdot T \\
v_{k} \\
\eta_{k}
\end{array}\right]
$$

A detailed description of the extended Kalman filter can be found in literature, e.g. in [10].

The first three elements of the Kalman Filter state vector are the desired vehicle state $\mathbf{x}$ for the map matching process. The corresponding upper left $3 \times 3$ sub-matrix of the Kalman filter state covariance matrix is the desired covariance matrix for map matching $\boldsymbol{\Sigma}_{\mathbf{x}}$.

The use of a vehicle model with very low dynamics is mainly justified by the lack of highly dynamic sensor data in our system. However, the system model may be easily extended to account for different sensor characteristics and application specific needs, e.g. dead reckoning using odometry for built-in systems.

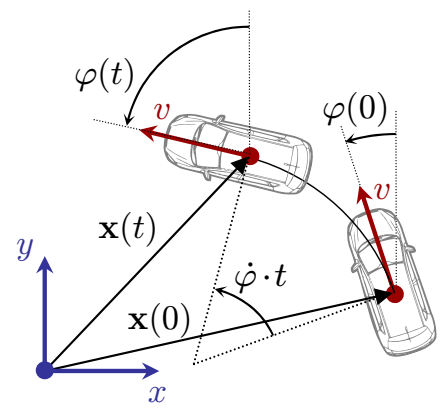

Fig. 1. Vehicular motion for constant velocity and yaw rate.

\section{ROAD NETWORK GEOMETRY}

Reconstruction of the road network from a given set of shape points is commonly done by linear interpolation of the shape points. Subsequent matching of the vehicle position to the interpolated road network typically yields position residuals in the range of a few meters. Heading residuals, however, can easily reach $45^{\circ}$ during intersection traversal.

This may lead to ambiguities or even false matchings in some situations. Figure 2(a) shows an example of such an ambiguous situation, where three positions in the map have the same distance and orientation difference to the assumed vehicle position. The ambiguity can easily be resolved by using higher-order interpolation as shown in figure 2(b).

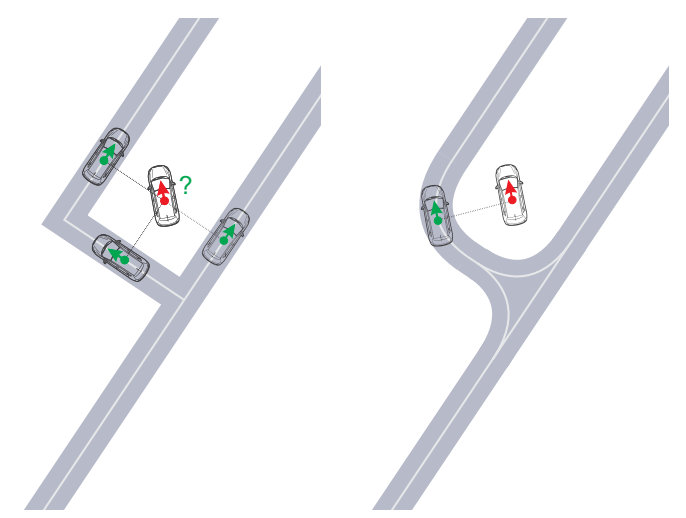

(a) Single position estimate.

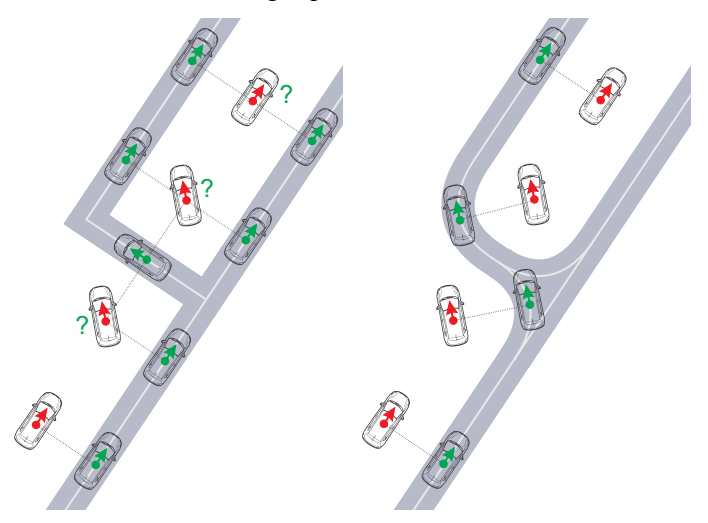

(b) Sequence of position estimates.

Fig. 2. Example of an ambiguous map matching situation. The red arrow denotes the vehicle position and heading estimate, the green arrows denote possible map matching results. Left image: linear interpolation, right image: cubic interpolation.

As figure 2(b) shows, the occurrence of such ambiguities has a direct impact on matching robustness. In case of linear interpolation, the given sequence of GPS positions results in two vehicle paths with the same probability. Higher-order interpolation would lead to a single matching result.

Unfortunately, current digital maps do not give any information about road curvature. Instead, we will exploit the knowledge that, according to e.g. the german road construction guidelines [4]), roads are typically built with smooth curvature change. Furthermore, we know that our road will run exactly through its starting node $\mathbf{p}_{0}$, the $n$ given shape points $\mathbf{p}_{k}, k=1 . . n$ and its ending node $\mathbf{p}_{n+1}$. 
Instead of linearly interpolating adjacent shape points, we will therefore use a cubic spline polynomial for every pair of adjacent shape points. Furthermore, we will require smooth curvature at all transitions between interpolation polynomials.

A nice way to assure that the interpolating polynomials run exactly through the given shape points is to use the Hermite formulation of the spline polynomial, where two of the four control points are just the desired starting and ending points (see e.g. [11] for a detailed explanation). For a given set of $n$ shape points $s_{i}, i=1, \ldots, n$, we get $n-1$ interpolation polynomials

$$
\begin{aligned}
& \mathbf{p}_{i}(\lambda)=\left(2 \lambda^{3}-3 \lambda^{2}+1\right) \cdot \mathbf{s}_{i}(0) \\
& +\left(-2 \lambda^{3}+3 \lambda^{2}\right) \quad \cdot \mathbf{s}_{i}(1) \\
& +\left(\lambda^{3}-2 \lambda^{2}+\lambda\right) \quad \cdot \quad \mathbf{s}_{i}^{\prime}(0) \\
& +\left(\lambda^{3}-\lambda^{2}\right) \cdot \mathbf{s}_{i}^{\prime}(1),
\end{aligned}
$$

where $\lambda \in[0,1]$.

Requiring $\mathcal{C}^{2}$ continuity delivers a system of $2 n-2$ equations for the unknown $2 n$ tangent vectors $\mathbf{s}_{i}^{\prime}(0)$ and $\mathbf{s}_{i}^{\prime}(1), i=1, \ldots, n$. Together with the natural spline condition $\mathbf{s}_{0}^{\prime \prime}(0)=0$ and $\mathbf{s}_{(n-1)}^{\prime \prime}(1)=0$, we get a tridiagonal system of linear equations that can be solved for the unknown tangents. An implementation of this solution can be found e.g. in [8].

The actual interpolation takes place in three steps (see figure 3). First, road elements that are likely to be smoothly connected in reality are combined to sequences of road elements. This is done based on heuristics like a small orientation difference between road elements or adjacent road elements having the same road class. Secondly, each sequence of road elements is interpolated according to the method described above, using the natural spline condition for both end points of the sequence. Finally, for every allowed transition between road elements that are not part of the same sequence, an additional interpolated road element is inserted in order to get a smooth transition for every possible maneuver.

To take into account the persisting position uncertainty of the road elements, the spline coordinates can additionally be modeled as random variables as described in [5].

\section{RoAd NETWORK TOPOLOGY}

Road network topology is represented by a stochastic finite state machine that is adopted directly from the digital map. Every edge in the map is represented by one state $k_{i}$ for each driving direction.

The non-zero state transition probabilities $p\left(k_{j} \mid k_{i}\right)$ follow directly from three possible driving maneuvers for any given road element:

- The vehicle proceeds to an adjacent road element.

- The vehicle remains on the current road element and keeps its driving direction. This is modeled by a loop in the state space.

- The vehicle remains on the current road element but changes driving direction (U-turn). This corresponds to a transition to the state denoting the opposite driving

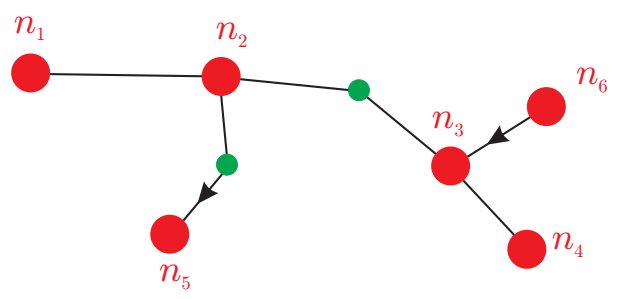

(a) Original road network with shape points.

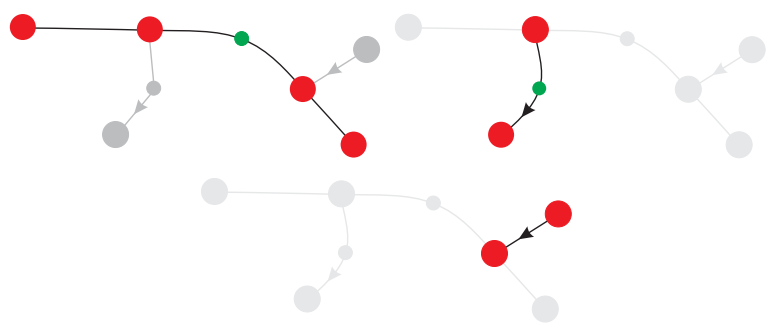

(b) Interpolation of connected sequences of road elements.

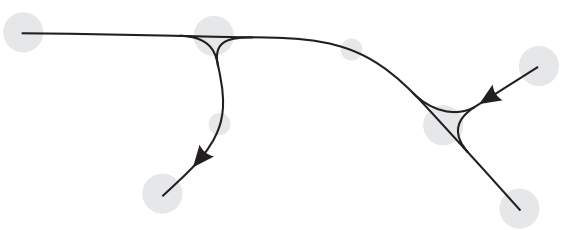

(c) Intersection interpolation.

Fig. 3. Illustration of the interpolation process for an example road network. Red dots denote road network nodes, green dots denote additional shape points. Interpolation is first done for sequences of road elements with small orientation differences, intersections are interpolated subsequently.

direction. This transition does not exist for one-way road elements.

The probability for the u-turn maneuver is denoted $p_{\text {turn }}$ and should be chosen very close to 0 . None of the remaining transitions is preferred, i.e. the remaining probability $p_{\text {trans }}=1-p_{\text {turn }}$ is split up equally to all other transitions, including the loop transition.

For a state $k_{i}$ with $n$ transitions to neighboring states $k_{j}$ (excluding the u-turn transition), each transition has the probability

$$
p\left(k_{j} \mid k_{i}\right)=p\left(k_{i} \mid k_{i}\right)=\frac{p_{\text {trans }}}{n+1} .
$$

An example representation for one road element with two allowed driving directions is given in figure 4. The state diagram for an example road network is given in figure 5.

\section{MAP MATCHING}

Similar to our previous work [6], map matching will be formulated as finding the most likely sequence of states in a Hidden Markov Model as introduced in section V. For a detailed introduction to Markov Models, see e.g. [3].

The emission probabilities for each state $k_{i}$ correspond to the probability $p\left(\mathbf{x} \mid k_{i}\right)$ that a certain measurement $\mathbf{x}$ is observed for a vehicle standing on that road element. The optimal path $\mathbf{i}=\left[i_{1}, i_{2}, \ldots, i_{T}\right]$ is now determined by maximizing the probability that a sequence of $T$ vehicle 

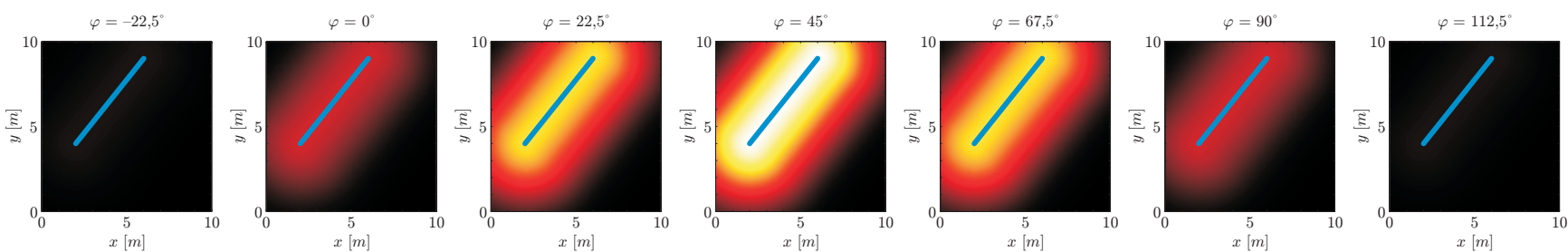

(a) Linear interpolation
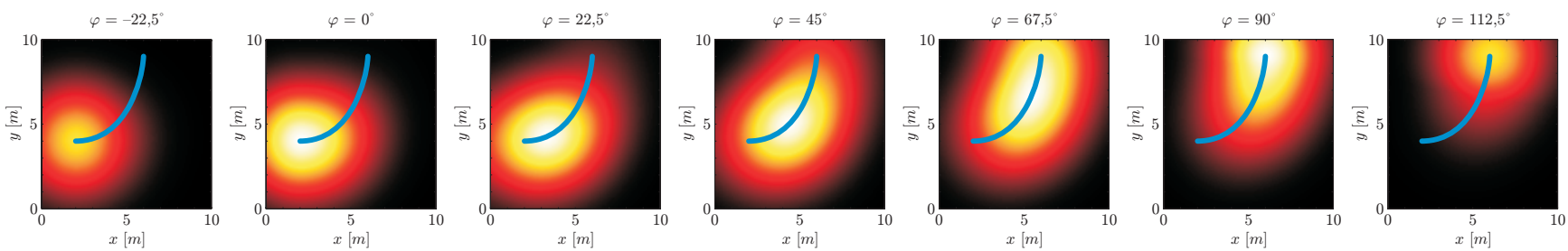

(b) Cubic interpolation

Fig. 6. Vehicle position likelihood for different vehicle orientations. The blue line denotes a road element with (a) constant orientation of $45^{\circ}$ and (b) an orientation changing from $0^{\circ}$ to $90^{\circ}$ respectively.

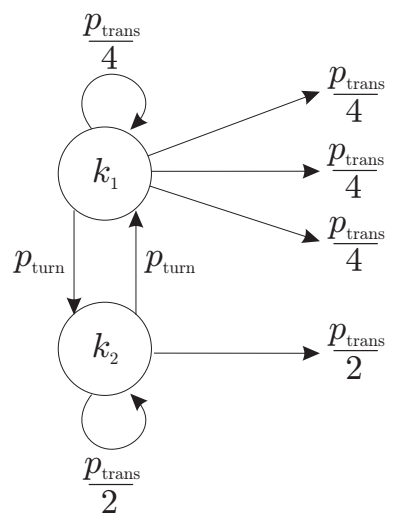

Fig. 4. Representation of a two-way road element as one state for each direction. All exiting transitions get the same probabilities, while the probabilities for u-turns and staying on the road element are chosen differently.

states $\mathbf{x}_{t}, t=1, \ldots, T$ belongs to a sequence of $T$ road elements $x_{i_{t}}$. With the transition probabilities $p\left(k_{j} \mid k_{i}\right)$ from section $\mathrm{V}$, this can be formulated using the chain rule as

$$
\hat{\mathbf{i}}=\underset{\mathbf{i}}{\operatorname{argmax}}\left(\prod_{t=2}^{T} p\left(\mathbf{x}_{t} \mid k_{i_{t}}\right) \cdot p\left(k_{i_{t}} \mid k_{i_{t-1}}\right)\right) \cdot p\left(\mathbf{x}_{1} \mid k_{i_{1}}\right) .
$$

Iterative computation of the optimum path can be done efficiently using the Viterbi algorithm.

The probability $p\left(\mathbf{x} \mid k_{i}\right)$ is modeled by the Mahalanobis distance of the measurement $\mathbf{x}$ and the road element $k_{i}$.

Using the interpolated representation of a particular road element, the Mahalanobis distance of a particular point $\mathbf{p}_{j}(\lambda)$ on the $j$-th interpolation interval to the vehicle state estimate $x$ is given by

$$
D_{i}(j, \lambda)=\sqrt{\mathbf{d}(i, j, \lambda)^{T} \cdot \boldsymbol{\Sigma}^{-1} \cdot \mathbf{d}(i, j, \lambda)}
$$

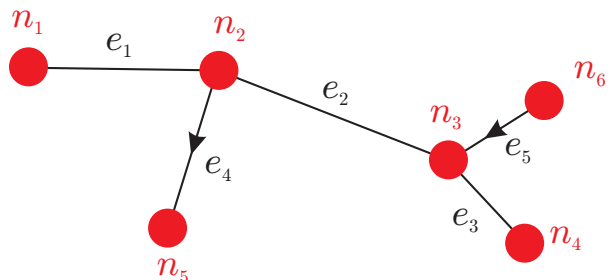

(a)

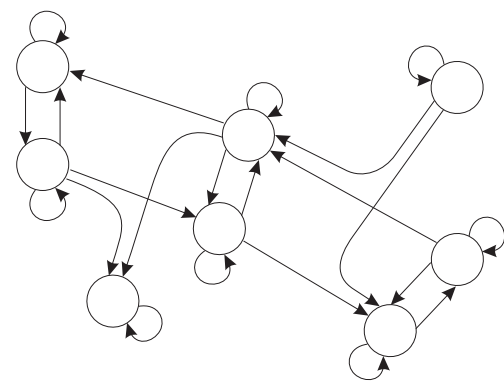

(b)

Fig. 5. Example road network and corresponding state representation. Oneway road elements are marked with a black arrow. Only state transitions with non-zero transition probability are shown.

with

$$
\mathbf{d}(i, j, \lambda)=\left[\begin{array}{c}
\mathbf{p}_{j}(\lambda) \\
\angle \mathbf{p}_{j}(\lambda)
\end{array}\right]-\mathbf{x} .
$$

The covariance matrix $\boldsymbol{\Sigma}$ has to take into account both vehicle state uncertainties $\boldsymbol{\Sigma}_{\mathbf{x}}$, which were already determined from the Kalman filter state covariance matrix, and map uncertainties $\boldsymbol{\Sigma}_{\mathbf{p}}$ which have to be chosen according to the precision of the given map.

The Mahalanobis distance for an entire road element is determined by finding the minimum distance to any point on that particular road element

$$
D_{i}=\min _{j, \lambda} D_{i}(j, \lambda) .
$$




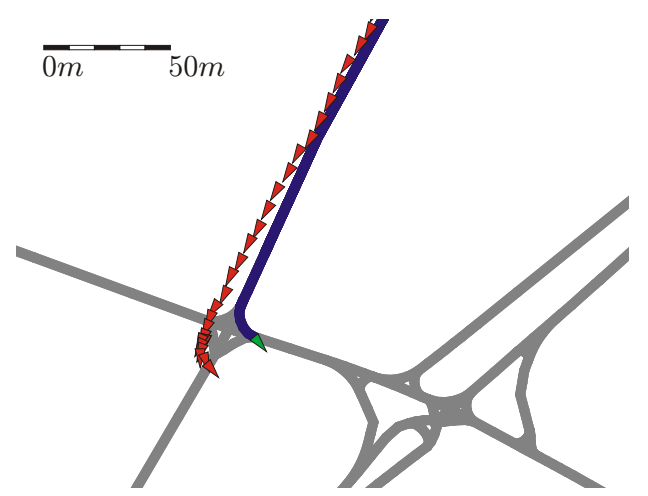

(a)

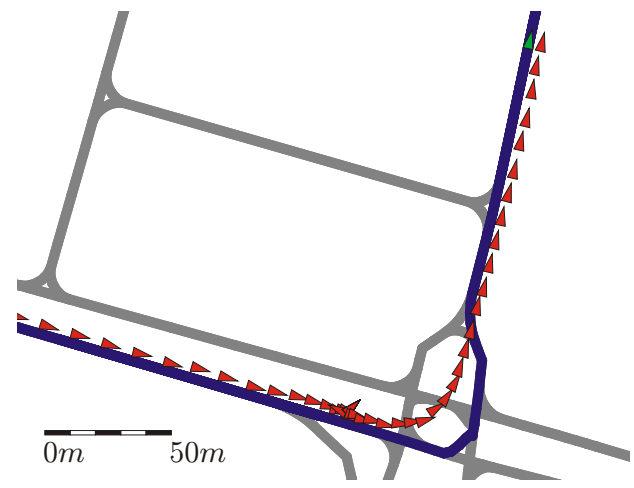

(c)

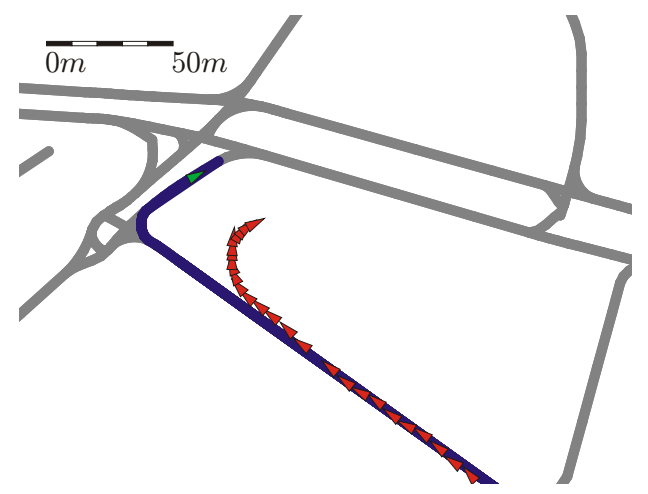

(b)

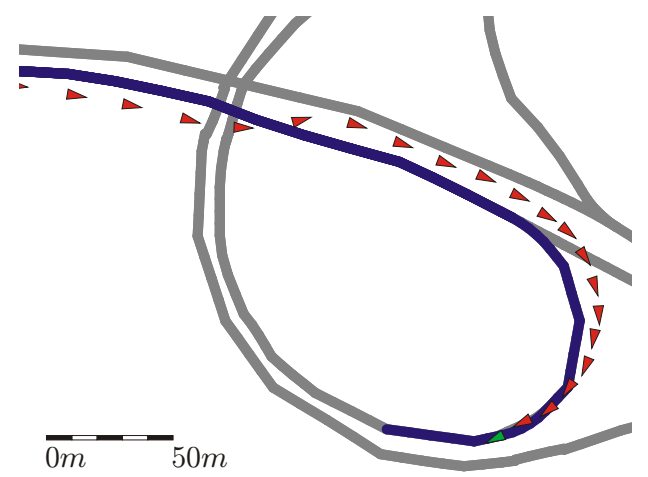

(d)

Fig. 7. Map matching results for typical inner-city scenes. The sequence of GPS positions is shown in red, the current map matched position is shown in green. The blue road elements denote the optimum path.

Figure 6 illustrates the resulting position measurement likelihood in comparison to the likelihood for linearly interpolated road elements as used in [6]. While the likelihoods for linear interpolation have no distinct maxima and decrease rapidly for increasing orientation difference, cubic interpolation delivers distinct maxima over a wide range of vehicle orientations.

\section{EXPERIMENTAL RESULTS}

The proposed map matching was tested in the inner city of Karlsruhe, Germany.

During the test run, several assignments of the vehicle position to the wrong road element due to GPS deviations or map errors occurred. However, the model compensated these single outliers and returned a correct estimation of the vehicle path history for the entire test run of more than one hour duration.

Figure 7 shows some example results from the test run. The GPS positions are shown in red, the optimum path estimate is shown in blue. The map matched position for the current time instant is shown in green.

The lateral offset in figure 7(a) is possibly caused by an offset GPS measurement. Figures 7(b) to 7(d) show errors due to imprecise map digitization. Errors like these are very typical for inner-city situations and led to position offsets of up to $40 \mathrm{~m}$ for a short period of time. In all cases, the map matching returns the correct path.
In order to evaluate the robustness of the map matching algorithm, the original GPS sequence was manually shifted by $100 \mathrm{~m}$ to a random direction. The original GPS positions are shown in figure $8(\mathrm{a})$. The map matching results are shown in figure 8(b) to 8(d) together with the corresponding shifted GPS measurements. At the beginning (figure 8(b)), the positions are matched to a wrong road with the same heading, but as soon as more GPS measurements have supported the correct vehicle path, the correct path is chosen (figure 8(c)). As figure 8(d) shows, the entire matched vehicle path matches the original GPS positions very well.

\section{CONCLUSION}

We presented a robust map matching method that uses a standard GPS receiver and an industry-standard digital map as only input data. Increased robustness compared to other methods was achieved by exploiting vehicular motion constraints in an extended Kalman filter and by interpolating the given road network using cubic splines. Representation of the road network topology in a hidden Markov model allows for reliable estimation of the vehicle position history and increases robustness further.

The proposed system was designed and tested with the smallest possible set of sensors in order to make it usable for a wide range of applications. The map matching allows for easy integration of further sensor information or using higher precision map data which is likely to improve map 


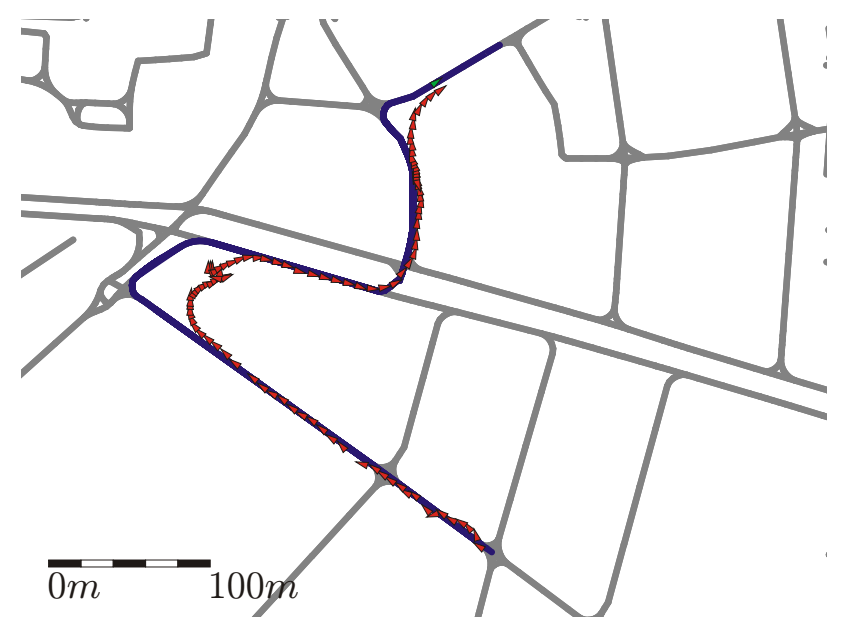

(a)

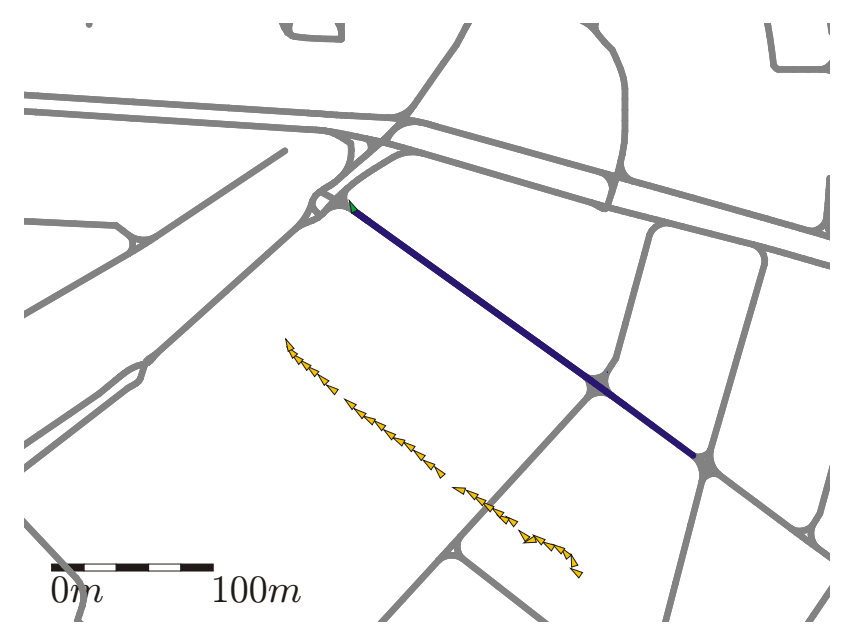

(c)

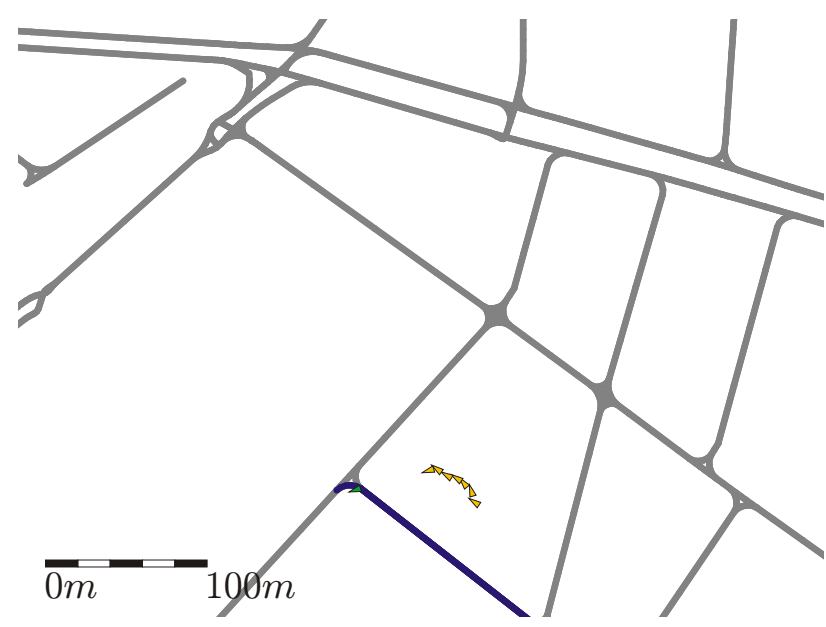

(b)

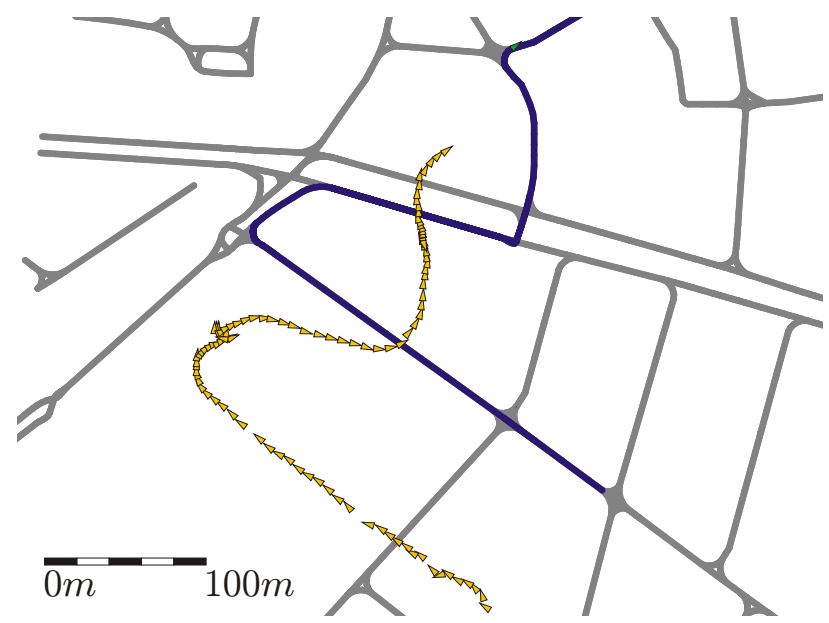

(d)

Fig. 8. Map matching results for a GPS position offset of $100 \mathrm{~m}$. (a) shows the original sequence of GPS positions (red) and the corresponding optimum path (blue). (b), (c) and (d) show the GPS positions with a constant position offset of $100 \mathrm{~m}$ (yellow) and the map matching results (blue) at different times. At the beginning, the road assignment is incorrect, but after a short while, the correct path is chosen.

matching results further.

Experimental results have shown that both the current vehicle positions and the position history are matched precisely. Even with severely disturbed GPS signals as the only input signals, the proposed method allows for robust map matching and reliable vehicle position history estimation.

\section{REFERENCES}

[1] David Bernstein and Alain Kornhauser. An introduction to map matching for personal navigation assistants. In The Transportation Research Board 77th Annual Meeting, Washington, D.C, 1996.

[2] Sotiris Brakatsoulas, Dieter Pfoser, Randall Salas, and Carola Wenk. On map-matching vehicle tracking data. pages 853-864, 2005.

[3] Richard O. Duda, Peter E. Hart, and David G. Stork. Pattern classification. Wiley, 2. edition, 2001.

[4] FGSV. RAS-L - Richtlinien für die Anlage von Straßen - Teil: Linienführung, 1995.

[5] Carsten Hasberg and Stefan Hensel. Online-estimation of road map elements using spline curves. In Proc. of the 11th IEEE International Conference on Information Fusion, 2008.

[6] B. Hummel. Dynamic and Mobile GIS: Investigating Changes in Space and Time, chapter Map Matching for Vehicle Guidance. CRC Press, 2006.
[7] International Organization for Standardization, Geneva. Intelligent transport systems - Geographic Data Files (GDF) - Overall data specification, 2004.

[8] William H. Press, Saul A. Teukolsky, William T. Vetterling, and Brian P. Flannery. Numerical recipes. Cambridge Univ. Press, 3. edition, 2007.

[9] Mohammed A. Quddus, Washington Yotto Ochieng, Lin Zhao, and Robert B. Noland. A general map matching algorithm for transport telematics applications. GPS Solutions, 7(3):157-167, December 2003.

[10] Greg Welch and Gary Bishop. An introduction to the Kalman Filter. 1995.

[11] Raymond Zavodnik and Herbert Kopp. Graphische Datenverarbeitung. Hanser, 1995. 